\title{
Repolarization of tumor infiltrating macrophages and increased survival in mouse primary CNS lymphomas after XPO1 and BTK inhibition
}

\author{
Isabel Jiménez $z^{1,2}$ - Júlia Carabia ${ }^{1,2} \cdot$ Sabela Bobillo ${ }^{2,3} \cdot$ Carles Palacio $^{2,3} \cdot$ Pau Abrisqueta ${ }^{2,3} \cdot$ Carlota Pagès $^{1,2}$. \\ Juan C. Nieto ${ }^{1,2}$. Josep Castellvi ${ }^{4} \cdot$ Francisco Martínez-Ricarte $^{5} \cdot$ Lourdes Escoda $^{6}$. Cristóbal Perla $^{7}$. \\ Dennis H. Céspedes Torrez ${ }^{7}$. Joan Boix ${ }^{1,2}$. Noelia Purroy ${ }^{1,2,10}$. Lluís Puigdefàbregas ${ }^{1,2}$. Joan Seoane ${ }^{2,8,9}$. \\ Francesc Bosch ${ }^{2,3} \cdot$ Marta Crespo ${ }^{1,2}$
}

Received: 20 March 2020 / Accepted: 2 July 2020 / Published online: 20 July 2020

(c) The Author(s) 2020

\begin{abstract}
Background Patients diagnosed with primary central nervous system lymphoma (PCNSL) often face dismal outcomes due to the limited availability of therapeutic options. PCNSL cells frequently have deregulated B-cell receptor (BCR) signaling, but clinical responses to its inhibition using ibrutinib have been brief. In this regard, blocking nuclear export by using selinexor, which covalently binds to XPO1, can also inhibit BCR signaling. Selinexor crosses the blood-brain barrier and was recently shown to have clinical activity in a patient with refractory diffuse large B-cell lymphoma in the CNS. We studied selinexor alone or in combination with ibrutinib in pre-clinical mouse models of PCNSL.

Methods Orthotopic xenograft models were established by injecting lymphoma cells into the brain parenchyma of athymic mice. Tumor growth was monitored by bioluminescence. Malignant cells and macrophages were studied by immunohistochemistry and flow cytometry.

Results Selinexor blocked tumor growth and prolonged survival in a bioluminescent mouse model, while its combination with ibrutinib further increased survival. CNS lymphoma in mice was infiltrated by tumor-promoting M2-like macrophages expressing PD-1 and SIRP $\alpha$. Interestingly, treatment with selinexor and ibrutinib favored an anti-tumoral immune response by shifting polarization toward inflammatory M1-like and diminishing PD-1 and SIRP $\alpha$ expression in the remaining tumorpromoting M2-like macrophages.

Conclusions These data highlight the pathogenic role of the innate immune microenvironment in PCNSL and provide preclinical evidence for the development of selinexor and ibrutinib as a new promising therapeutic option with cytotoxic and immunomodulatory potential.
\end{abstract}

Keywords PCNSL $\cdot \mathrm{XPO} 1 \cdot \mathrm{BTK} \cdot$ Innate immune system

$\begin{array}{ll}\text { Abbreviations } \\ \text { PCNSL } & \begin{array}{l}\text { Primary central nervous system } \\ \text { lymphoma }\end{array} \\ \text { BCR } & \text { B-cell receptor }\end{array}$

Isabel Jiménez, Júlia Carabia and Francesc Bosch, Marta Crespo have contributed equally to this work

Electronic supplementary material The online version of this article (https://doi.org/10.1007/s11060-020-03580-y) contains supplementary material, which is available to authorized users.

Marta Crespo

macrespo@vhio.net

Extended author information available on the last page of the article
NHL Non-Hodgkin lymphoma

ABC-DLBCL Activated B-cell diffuse large B-cell lymphoma

GCB-DLBCL Germinal center B-cell diffuse large B-cell lymphoma

SINE Selective inhibitor of nuclear export

BBB Blood-brain barrier

BLI Bioluminescence imaging

PDX Patient derived xenograft

NSG NOD-SCID- $\gamma$ : non-obese diabetic- severe combined immune deficiency- interleukin 2 receptor gamma chain

IHC Immunohistochemistry

SEM Standard error of the mean 


$\begin{array}{ll}\text { COO } & \text { Cell of origin } \\ \text { CLL } & \text { Chronic lymphocytic leukemia } \\ \text { TAM } & \text { Tumor-associated macrophages } \\ \text { M-CSF } & \text { Macrophage colony-stimulating factor } \\ \text { IDO } & \text { Indoleamine 2, 3 dioxygenase } \\ \text { MRI } & \text { Magnetic resonance imaging } \\ \text { CI } & \text { Combination index }\end{array}$

\section{Background}

Primary central nervous system lymphoma (PCNSL) is a rare and aggressive non-Hodgkin lymphoma (NHL) localized to the CNS in the absence of systemic involvement that represents around $4 \%$ of all brain tumors and 4 to $6 \%$ of all extranodal lymphomas [1]. Approximately $95 \%$ of PCNSL are classified as activated B-cell diffuse large B-cell lymphoma (ABC-DLBCL) based on histopathology, gene expression and mutational landscape [2]. Current treatment options for PCNSL include high doses of chemotherapy able to cross the blood-brain barrier (BBB) combined with anti-CD20 monoclonal antibodies and the addition of whole brain radiation in some settings; also, autologous stem cell transplantation is considered for young patients. Patients diagnosed with PCNSL respond poorly to the available treatments and often face dismal outcomes, especially in the relapsed setting, with an estimated overall survival of $30 \%$ at 5 years [3]. This notion of the poor prognosis of PCNSL can be explained by particular biological characteristics of the tumor. First, PCNSL are characterized by a high frequency of concomitant MYD88 and CD79B mutations [4] along with lesions related to B-cell development and function (e.g. BLIMP1), and the NF- $\mathrm{KB}$ pathway (e.g. CARD11 or TBL1XR1). The involvement of the BCR signaling in PCNSL has prompted the use of the BTK inhibitor ibrutinib, that, although it can cross the BBB [5], achieves wide but short duration responses [6-8]. In addition, PCNSL develop in a special microenvironment of unique immune surveillance, which could contribute to an inefficient response of the immune system against lymphoma cells. In this regard, the few reports examining the tumor-infiltrating immune microenvironment show that it is mainly composed by macrophages and by T-cells to a lesser extent [9-13]. Also, an intriguing high proportion of PCNSL have genetic lesions that potentially avoid being recognized by T-cells, namely HLA loses and PD-L1/2 amplifications found in up to $80 \%$ of patients [14]. Finally, the poor prognosis can also be explained by the diminished capacity of some drugs to cross the BBB. Selinexor (KPT-330), a BBB permeable small molecule [15], is a Selective Inhibitor of Nuclear Export (SINE) compound that binds to the cargo binding pocket of XPO1 (exportin-1/CRM1) and inhibits its activity. This results in the nuclear accumulation of tumor suppressor proteins and cell cycle regulators together with the activation of tumor suppressor proteins, which translates in cell cycle arrest and specific anti-cancer activity across a wide range of hematological and solid malignancies [16]. In July 2019, selinexor was approved by the FDA to treat patients with multiple myeloma while in May 2020 it was approved for systemic relapsed/refractory DLBCL after positive results in a phase IIb trial [17]. Also, the ability of selinexor to inhibit both the BCR and the NF- $\mathrm{KB}$ signaling pathways makes this drug interesting for studies in NHL [16, 18]. Recently, in a clinical case study, selinexor was reported to inhibit refractory DLBCL with CNS involvement [19]. In order to provide a pre-clinical rationale for the design of new therapeutic strategies for patients diagnosed with PCNSL, herein we evaluate the role of XPO1 and BTK inhibition in intracerebral xenograft murine models, focusing on malignant cells and the innate immune microenvironment.

\section{Materials and methods}

\section{In vivo modeling of PCNSL}

All animal experiments were approved by the local Ethical Committee for the Use of Experimental Animals. Detailed methods including treatment schedules can be found in Supplementary information. Briefly, brains of eight-week-old athymic female mice were injected with OCI-Ly10 cells stably transfected with luciferase, as previously reported [20]. Tumor growth was monitored by bioluminescence imaging (BLI) using IVIS® Spectrum system and Living Image software (PerkinElmer).

Patient derived xenograft (PDX) model was established by intracerebral injection of human lymphoma cells isolated from a brain biopsy in eight-week-old NOD-SCID- $\gamma$ (NSG) female mice. Next, expanded CD19 ${ }^{+}$tumor cells were inoculated into the brain parenchyma of eight-weekold athymic female mice as specified above. Human tumor sample was obtained from a patient diagnosed with PCNSL at Hospital Universitari Joan XIII, Tarragona (Spain) after approval from the local Clinical Research Ethics Committee according to the principles of the Declaration of Helsinki and obtaining written informed consent from the patient.

\section{Flow cytometry and immunohistochemistry (IHC) analysis}

Mice brains were collected in cold RPMI-1640 medium immediately after euthanasia and the two hemispheres were separated with a razor blade. One hemisphere was used for IHC and the other one was processed for flow cytometry. Detailed methods can be found in Supplementary information. 


\section{Statistical analysis}

Results are expressed as the mean \pm standard error of the mean (SEM) of at least four independent experiments or subjects. The statistically significant differences between groups were analyzed using the Mann-Whitney test or one or two-way ANOVA, and $\mathrm{P}<0.05$ was considered significant. Detailed methods can be found in Supplementary information.

\section{Results}

\section{DLBCL cell lines have equivalent sensitivity to selinexor regardless of their cell of origin (COO)}

ABC-DLBCL relies heavily on NF- $\mathrm{KB}$ signaling and shows chronic BCR activation that is needed for survival, which translates into differential sensitivity to drugs targeting these pathways between ABC and GCB DLBCL cases [21, 22]. Since increased expression of $X P O 1$ has been related to resistance to chemotherapy and worse prognosis in different neoplasias [23], we studied the potential relationship between expression of XPO1 and sensitivity to selinexor in DLBCL cell lines. Although mRNA expression of XPO1 was significantly higher in ABC-DLBCL cell lines (Fig. 1a), we did not find differential in vitro sensitivity to selinexor according to $\mathrm{COO}$ (Fig. 1b, c). Finally, we interrogated the publicly available data on gene expression of primary DLBCL cases [24] and we did not observe any association between the $\mathrm{COO}$ and the expression of XPO1 (Fig. 1d).

\section{Selinexor blocks tumor growth and prolongs survival in a bioluminescent orthotopic mouse model of PCNSL.}

We next assessed the role of XPO1 inhibition in PCNSL using an intracerebral orthotopic xenograft murine model established by stereotactic injection of the luciferaseexpressing OCI-Ly10 cell line into the cerebral parenchyma of nude athymic mice. OCI-Ly10 cell line was selected because it is derived from a patient diagnosed with $\mathrm{ABC}$ DLBCL and its genetic profile includes mutations in MYD88 (L265P) and CD79A (c. 4275_4316del) genes [20] (further verified in house), frequent in PCNSL [4]. Additionally, OCI-Ly 10 cells have successfully been used before in a PCNSL xenograft model in athymic mice for pre-clinical studies [20]. Tumoral growth was monitored using IVISSpectrum bioluminescence measurement. Eleven days after the injection of cells, all animals had developed detectable tumors restricted to the CNS and were randomly distributed into treatment or vehicle experimental groups (vehicle: $n=8$, mean radiance $=1.16 \cdot 10^{7} \mathrm{ph} / \mathrm{s} \pm 0.615 \cdot 10^{7} ;$ treatment: $\mathrm{n}=9$, mean radiance $\left.=2.32 \cdot 10^{7} \mathrm{ph} / \mathrm{s} \pm 1.86 \cdot 10^{7}\right)$. Mice were dosed with $5 \mathrm{mg} / \mathrm{kg}$ of selinexor or vehicle via oral gavage three times a week and subsequently, in order to non-invasively monitor the tumor growth, bioluminescence was assessed twice a week (Fig. 1e). Dose was selected based on previous pre-clinical data in mouse models of different neoplasias [25]. Treated mice showed a significantly slower increase in bioluminescence signal along time (two-way ANOVA: $p=0.0002$; Fig. 1f) indicating that the treatment with selinexor was able to notably slow down tumor growth. Specific time-point analysis showed that differences were significant as soon as 12 days after start of treatment (day 23 after injection: vehicle mean radiance $2.61 \cdot 10^{8} \mathrm{ph} / \mathrm{s} \pm 8.64 \cdot 10^{7}$ vs. $3.73 \cdot 10^{7} \mathrm{ph} / \mathrm{s} \pm 1.9 \cdot 10^{7}$ in selinexor; $\mathrm{p}=0.011$ ) while differences peaked at day 20 after treatment (day 31 after injection: $8.98 \cdot 10^{8} \mathrm{ph} / \mathrm{s} \pm 3.13 \cdot 10^{8}$ in vehicle vs. $1.19 \cdot 10^{8}$ $\mathrm{ph} / \mathrm{s} \pm 5.58 \cdot 10^{7}$ in selinexor group; $\mathrm{p}=0.0037$; Fig. 1f, representative cases can be seen in Fig. 1h). The blockage of intracerebral lymphoma growth induced by selinexor translated into a significantly increased survival, with a median survival of 48 days in the treatment group compared to 34 days in the vehicle group $(\mathrm{p}<0.0001$; Fig. $1 \mathrm{~g})$. At final point, histopathological analysis showed multifocal and infiltrative tumors affecting cerebral parenchyma and meninges of both cerebral hemispheres. Cells were highly proliferative (Ki-67 100\%), CD20-positive and were often found in the perivascular space resembling human PCNSL histology. Remarkably, infiltration was observed in both hemispheres, showing no preference for the right hemisphere, where the original inoculation of malignant cells was performed. Also, we did not observe variations in CD20 intensity among mice or within different areas of the same brain (representative cases shown at Fig. 1i and Supplemental Figure S1).

\section{The combination of selinexor and ibrutinib synergizes in vitro in DLBCL cell lines and increases survival of mice with CNS lymphoma}

The high frequency of molecular alterations in components of the BCR pathway can in part explain the response to BCR inhibitors in PCNSL. In this regard, ibrutinib in monotherapy in patients diagnosed with relapsed or refractory PCNSL achieves higher response rates compared to systemic DLBCL, however, the duration of the response is brief [5-7]. Alongside this, SINE compounds have also been shown to inhibit BCR signaling by downregulating the protein expression of BTK via enforced IKB nuclear retention in primary cells from patients with chronic lymphocytic leukemia (CLL) [16]. Moreover, the combination of selinexor and ibrutinib has shown in vitro synergism in CLL cells [18]. Accordingly, we observed reduced BCR signaling after treatment of OCI-Ly10 cells with selinexor and ibrutinib (Supplementary Figure S2A), as well as reduced BTK 

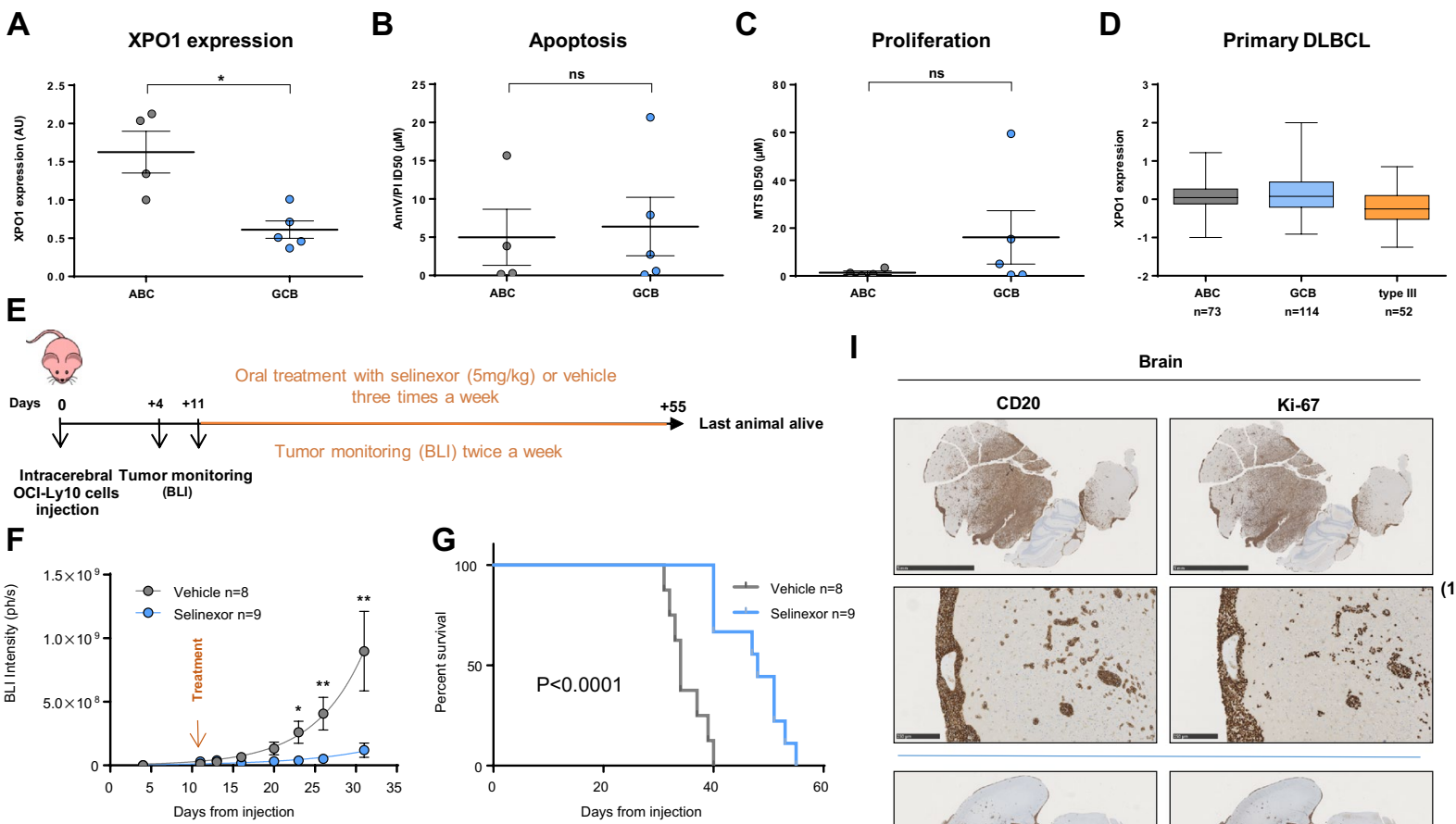

I

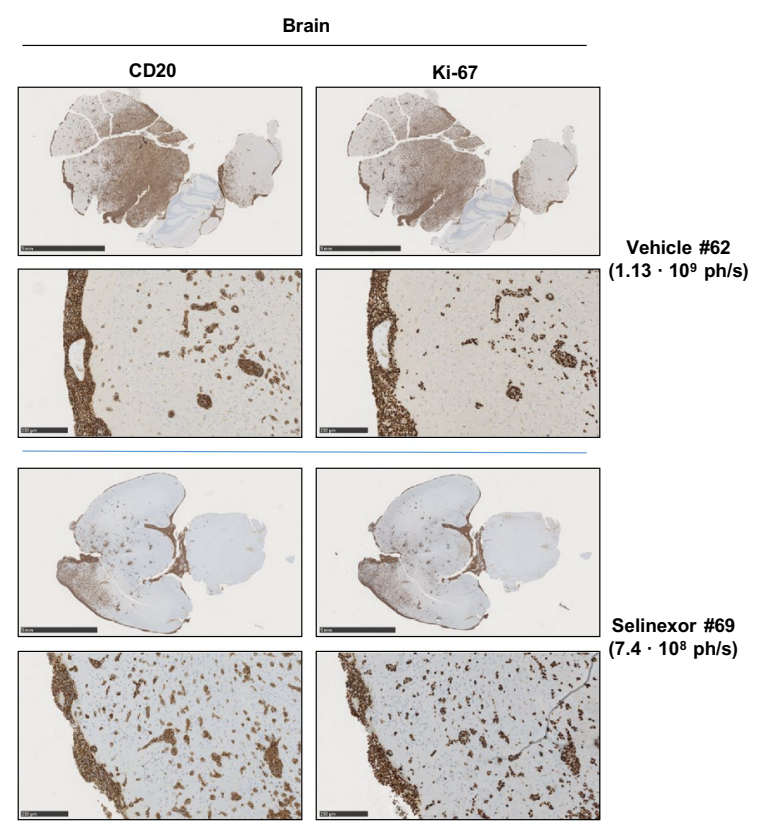

H

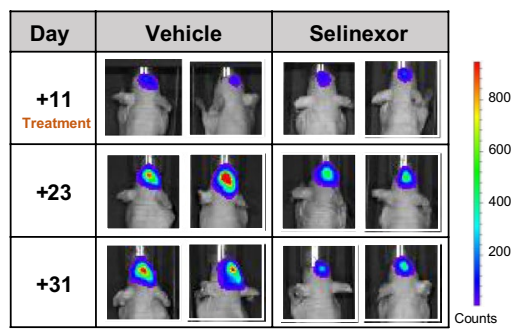
$\stackrel{+55}{\rightarrow}$ Last animal alive

Fig. 1 In vitro and in vivo effects of selinexor in PCNSL models. a XPO1 relative expression by QRT-PCR. Cells were treated with increasing doses of selinexor or vehicle (1\% DMSO) for $96 \mathrm{~h}$ and viability and proliferation was determined by Annexin-V-PI exclusion (b) or MTS method (c). d Relative XPO1 expression in DLBCL patients, using public data from ref [24]. e Scheme representing mice treatment and monitoring. $\mathbf{f}$ Tumor size as measured by BLI in mice treated with vehicle $(n=8)$ or selinexor $(n=9)$. Data is shown until day 31, last day when all animals were still alive. Two-way ANOVA analysis $(\mathrm{P}=0.0002)$. Asterisks indicate the result of Mann-Whitney

expression after $48 \mathrm{~h}$ of treatment with selinexor (Supplementary Figure S2B). Against this background, we hypothesized that combining XPO1 and BTK inhibition in PCNSL would have a synergistic therapeutic effect in our models. Firstly we treated a panel of cell lines in vitro with increasing doses of both drugs and analyzed apoptosis after $96 \mathrm{~h}$. In three out of four ABC-DLBCL cell lines we observed a strong synergism between the two compounds (Supplementary Figure S2C); remarkably, treatment with selinexor sensitized GCB-SUDHL4 cells to ibrutinib, as shown by the combination index values indicating strong synergism test at different time points. $(* \mathrm{P}<0.05, * * \mathrm{P}<0.01, * * * \mathrm{P}<0.001$. Graphs show mean \pm SEM) (g) Survival curves and (h) representative BLI images of the CNS tumors. i IHC analysis showing expression of CD20 and Ki-67 in representative mice brain parenchyma and meninges. The bars represent $5 \mathrm{~mm}$ in top panels and $250 \mu \mathrm{m}$ in bottom panels. ID50: inhibitory dose 50. ABC: activated-B cell. GCB: germinal center B-cell. BLI: bioluminescence imaging. $\mathrm{Ph} / \mathrm{s}$ : photons per second. ( $* \mathrm{P}<0.05$, $* * \mathrm{P}<0.01$, Mann-Whitney test. Graphs show mean $\pm \mathrm{SEM}$ )

between the two drugs (CI) (Supplementary Figure S2C, right panel).

We next sought to elucidate whether the synergy observed in vitro could be translated in vivo. Importantly, while ibrutinib is mainly metabolized by cytochrome P450, the metabolism of selinexor is independent of it, therefore it is unlikely that their co-administration could result in any effects on the exposure for the other drug [25, 26]. By using the same animal model described above, mice were distributed into the following four groups and started therapy 11 days after intracerebral injection of lymphoma cells: 
selinexor monotherapy $(5 \mathrm{mg} / \mathrm{kg}$ twice a week via oral gavage, $\mathrm{n}=12$, mean radiance $=3.95 \cdot 10^{6} \mathrm{ph} / \mathrm{s}$ ), ibrutinib monotherapy $(25 \mathrm{mg} / \mathrm{kg}$ daily in drinking water, $\mathrm{n}=9$, mean radiance $\left.=1.02 \cdot 10^{7} \mathrm{ph} / \mathrm{s}\right)$, combination therapy $(\mathrm{n}=11$, mean radiance $\left.=1.02 \cdot 10^{7} \mathrm{ph} / \mathrm{s}\right)$ and vehicle $(\mathrm{n}=9$, mean radiance $=3.21 \cdot 10^{6} \mathrm{ph} / \mathrm{s}$ ). Selinexor dose was adjusted (from three times a week to twice a week) in order to prevent potential toxicity of the drug combination, while ibrutinib dose was based on previous experience in CLL preclinical models [27] (Fig. 2a). Compared to vehicle, all three treatment regimens induced an equivalent significant effect in tumor growth kinetics in terms of decreased growth rate (Fig. 2b and c). Interestingly, the combination increased the survival of mice compared to vehicle, whereas there was no significant difference between ibrutinib and selinexor alone. Although the median survival increased up to 55 days, the survival curve of the mice treated with the combination was not statistically different from the ones from mice treated with the individual treatments (median survival of mice treated with vehicle: 35 days vs. survival for mice treated with selinexor: 40 days, $\mathrm{p}=0.001$; vehicle vs. ibrutinib, 43 days, $p=0.0005$; vehicle vs. combination, 55 days, $\mathrm{p}=0.0001$; Fig. 2d).

\section{CNS lymphoma is infiltrated by tumor-promoting M2-like macrophages expressing PD-1 and SIRPa}

Analysis of the tumor-infiltrating immune microenvironment has shown that tumoral cells in PCNSL are accompanied by tumor-associated macrophages (TAMs) and T-cells to less extend, which is related to bad prognosis. [9-13] Remarkably, TAMs in mouse and human colorectal cancer have been recently described to express the immune checkpoint PD-1 and to recover their potential to phagocyte tumoral cells when PD-1 is blocked [28]. To conduct an interactive study of the infiltrating innate immune cells and PCNSL, we inoculated OCI-Ly10 cells into the brain parenchyma of nude athymic mice, an experimental in vivo model that has been previously successfully used to study the modulation of the innate immune response against PCNSL [20, 29]. Brains were harvested after 24 days of cell injection and further processed for subsequent analysis. Histopathological analysis showed that tumors encompassing both cerebral hemispheres were infiltrated by macrophages expressing the surface glycoprotein F4/80, mainly in the meninges but also in the cerebral parenchyma; notably, F4/80-positive macrophages

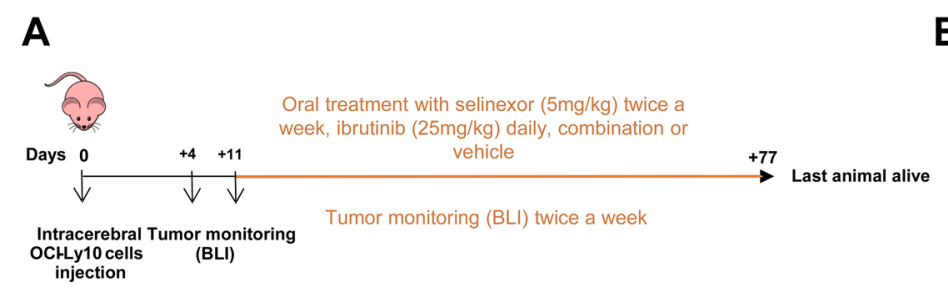

B

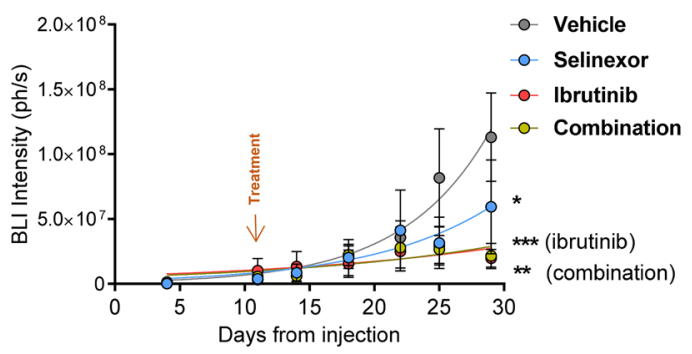

C

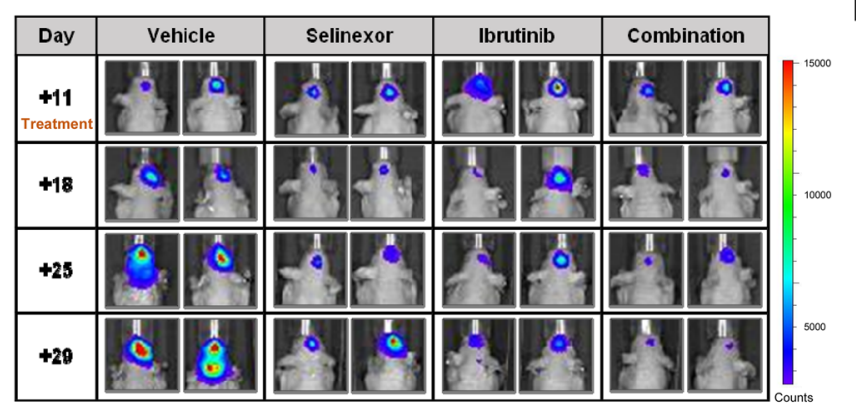

D

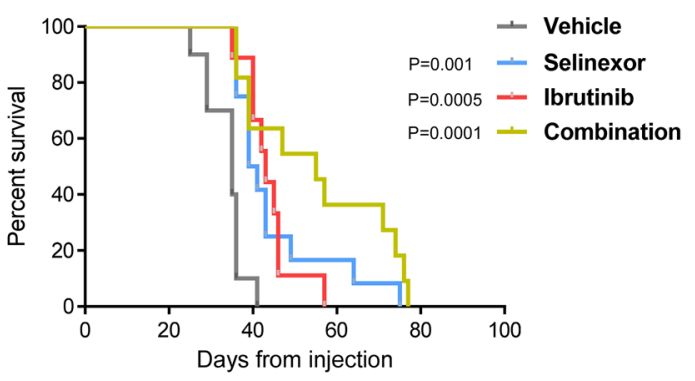

\begin{tabular}{|cccc|} 
& HR & $\mathbf{9 5 \%} \mathbf{C l}$ & p-value \\
\hline V vs. S & 3.064 & $2.7-21.22$ & 0.001 \\
V vs. I & 3.4 & $3.4-27.9$ & 0.0005 \\
V vs. Comb & 3.63 & $4.2-35.6$ & 0.0001 \\
S vs. Comb & 1.82 & $0.93-5$ & 0.1 \\
I vs. Comb & 2.031 & $1-7.3$ & 0.06
\end{tabular}

Fig. 2 Treatment with selinexor and ibrutinib further increases survival of mice with CNS lymphoma. a Scheme representing mice treatment and monitoring. b Tumor size as measured by BLI intensity. Data is shown until day 29, last day when all animals were still alive. $(* \mathrm{P}<0.05, * * \mathrm{P}<0.01, * * * \mathrm{P}<0.001$, Mann-Whitney test.
Graphs show mean \pm SEM). c Representative BLI images in mice from every treatment arm. d Survival curves of mice in the four treatment groups. Survival curves were generated using the Kaplan and Meier method, and statistically compared by the log-rank test. $H R$ hazard ratio, $C I$ confidence interval, $B L I$ bioluminescence imaging 
were completely absent in the areas of the brain that were not invaded by tumoral cells (Figs. 3a and Supplemental figure S1) as well as in healthy brains from control mice (Fig. 3a). Iba-1 staining further identified microglial cells and TAMs, which showed an amoeboid morphology when interacting with tumoral cells, consistent with an active state (Fig. 3a) [30]. TAMs can be polarized towards a pro-inflammatory (M1) or a tumor-promoting (M2) state, depending on microenvironment and external stimuli [31]. By flow cytometry, we analyzed the proportion of M1 and M2 TAMs and their expression of immune checkpoints in brains from mice with PCNSL. First, we observed that TAMs were evenly distributed between M1 and M2 (Fig. 3c). Of note, TAMs expressed PD-1, mainly the tumor promoting M2 subset (Fig. 3d). This suggests that the direct interaction of M2 macrophages with the tumor triggers the upregulation of PD-1 and thus impairs their phagocytic capacity, as has been recently discovered in an analogous role to tumor-infiltrating T-cells using both immunocompetent syngeneic and athymic xenograft mouse models [28]. SIRP $\alpha$ is a well described regulatory checkpoint on macrophages, its interaction with CD47 on malignant cells hampering the phagocytosis by macrophages [32]. Herein we observed that SIRP $\alpha$ was also preferentially expressed by M2 TAMs (Fig. 3e) and that the co-expression of PD-1 and SIRP $\alpha$ was also higher in the M2 subset (Fig. 3f), pointing out towards a severe inhibition of macrophage activity in CNSL.

The response of the innate immune system to PCNSL cells derived from a patient was further analyzed. For that, we developed an orthotopic PDX model using NSG mice to initially expand the freshly obtained primary malignant cells, as previously described by Rubenstein et al. and following the detailed protocol described in Supplementary methods [33] Next, we inoculated $2 \cdot 10^{5}$ lymphoma cells into the brain parenchyma of nude athymic mice [34]. Since the median survival of this mouse model was 22 days, infiltration by immune cells was analyzed after 18 days of tumor injection allowing infiltration by innate immune cells. In this model, TAMs were also found only amongst tumoral cells (Figs. 4a and Supplemental Figure S3) as assessed by IHC. TAMs from the PDX model displayed an immunophenotypic profile resembling the one found in TAMs from the cell line xenograft model. Along this line, a similar proportion of M1 and M2 (Fig. 4b) and a more frequent expression of PD-1 and SIRP $\alpha$ in M2 tumor-promoting macrophages was observed (Fig. 4c, d, e). In contrast to the OCI-Ly10 model, patient-derived PCNSL cells did express the SIRP $\alpha$ ligand CD47 (97.61\% of CD20 cells \pm 0.62 ).
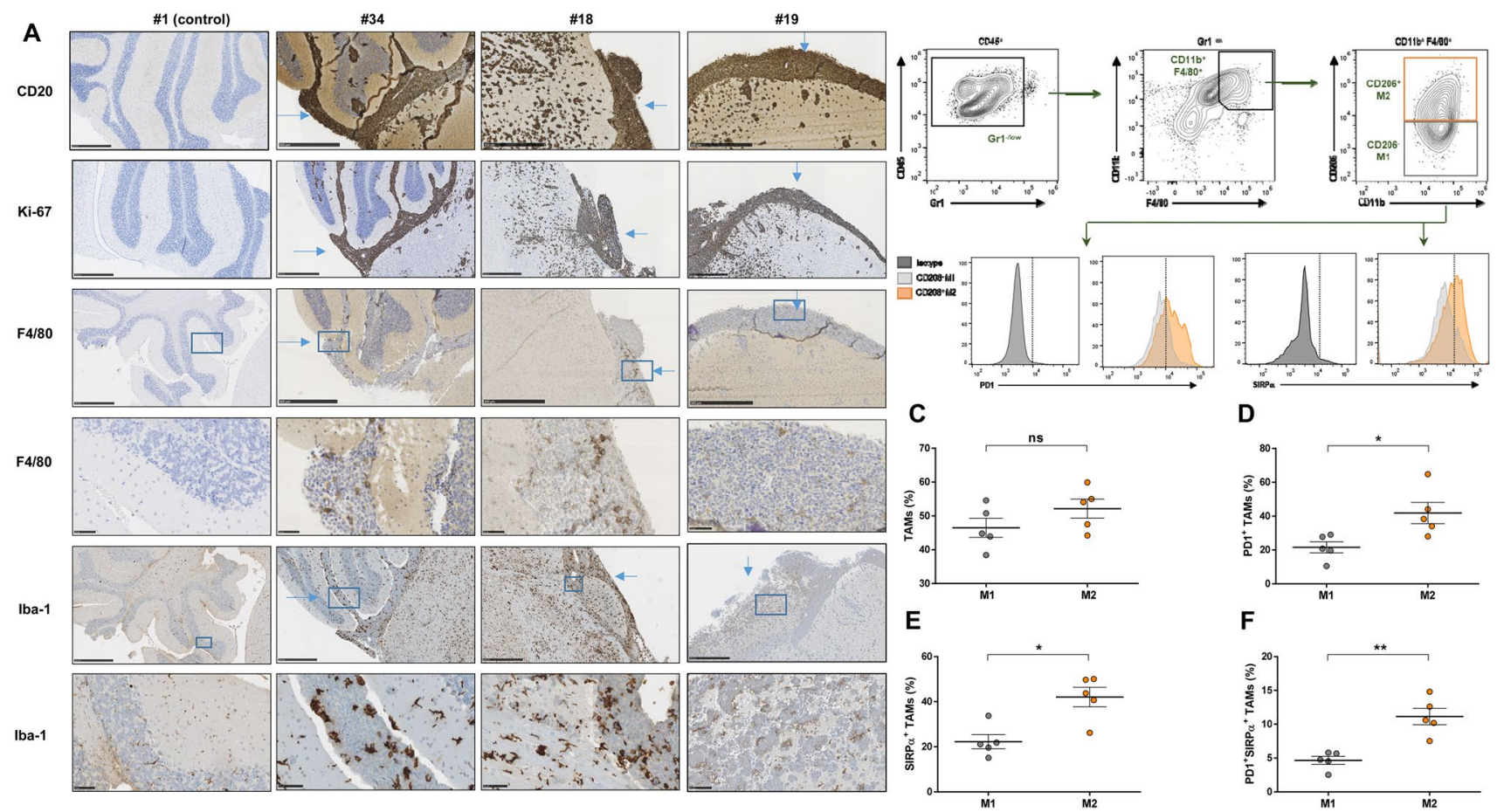

D

Fig. 3 OCI-Ly10 CNS lymphomas are infiltrated by innate immune cells. a Representative IHC images from brains obtained from three mice inoculated with OCI-Ly10 cells ( 24 days after injection). The bar represents $500 \mu \mathrm{m}$, except for fourth and last rows $(50 \mu \mathrm{m})$. b Gat- ing strategy for the analysis of TAMs. Percentage of macrophages (M1/M2) (c) expressing PD-1 (d), SIRP $\alpha$ (e) and co-expressing both (f) 


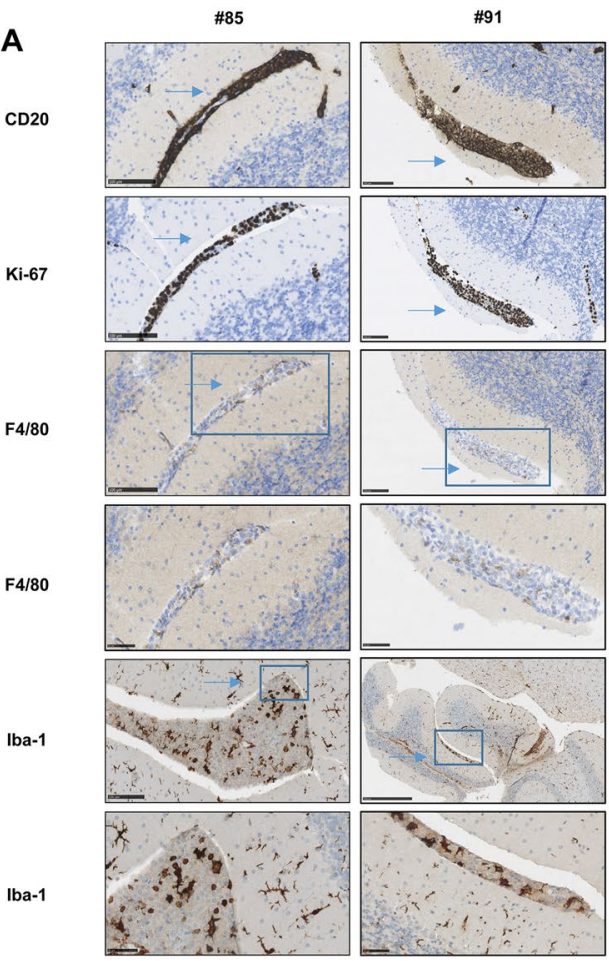

B

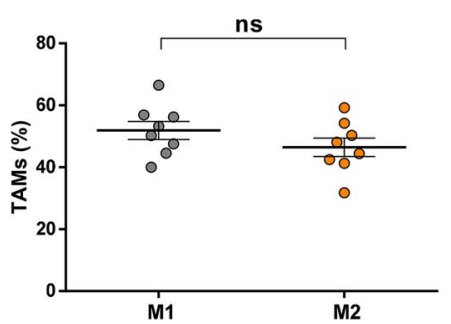

D

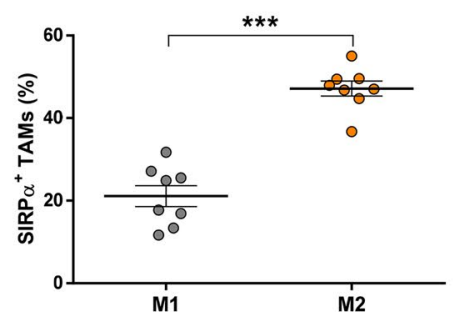

C

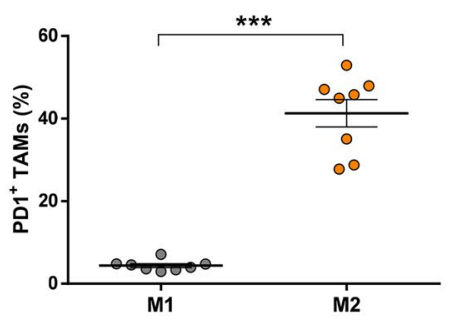

E

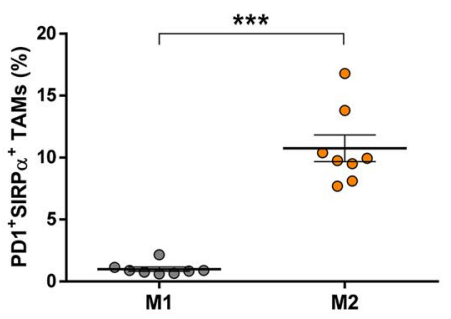

Fig. 4 PDX CNS lymphomas are infiltrated by innate immune cells. a Representative IHC images from brains obtained from two mice inoculated with patient-derived PCNSL cells (18 days after injection). The bar represents $100 \mu \mathrm{m}$ except for the four last rows $(50 \mu \mathrm{m})$. Per-

\section{Treatment with selinexor and ibrutinib favors TAM polarization toward pro-inflammatory M1-like and diminishes PD-1 and SIRPa expression in M2-like TAMs}

BTK protein has been shown to be crucial for tumor-promoting function of macrophages in different neoplasias, especially in CLL, where modulation of TAMs has been shown to be also a relevant mode of action of ibrutinib [35, 36]. Therefore, after showing that the combination of selinexor and ibrutinib restrains tumor growth and prolongs mice survival, and since both drugs are able to inhibit BTK, we hypothesized that these drugs could also cooperate to modify the innate immune response in PCNSL. In this regard, pre-clinical PCNSL models have previously demonstrated how immunomodulating drugs are able to shift macrophages polarization as well as have direct antitumoral effect. [20, 29] To test that, we treated mice bearing OCI-Ly10-CNS lymphomas with selinexor $5 \mathrm{mg} / \mathrm{kg}$ twice a week, ibrutinib $25 \mathrm{mg} / \mathrm{kg}$ daily or the combination of the two drugs for two weeks by oral gavage (Fig. 5a). We observed that selinexor and the combination shifted the M1/M2 ratio towards predominance of anti-tumoral M1 (Fig. 5b). Interestingly, while none of the individual treatments induced significant changes in the frequency of PD-1 or SIRP $\alpha$-positive M2 centage of macrophages (M1/M2) (b) expressing PD-1 (c), SIRP $\alpha$ (d) and co-expressing both (e). $(* \mathrm{P}<0.05, * * \mathrm{P}<0.01, * * * \mathrm{P}<0.001$, Mann-Whitney test. Graphs show mean \pm SEM)

macrophages, the drug combination significantly reduced the frequency of PD-1-positive, SIRP $\alpha$-positive (Fig. 5c, d, e) and double-positive M2 macrophages (Fig. 5f). In agreement the (CI) that the reduction of the expression of PD-1, SIRP $\alpha$ and their co-expression was synergistic $(\mathrm{CI}<1)$. This was accompanied by a reduction in PD-L1-expressing malignant cells (Fig. 5g, h) that was attributable to ibrutinib action since it was also observed under ibrutinib monotherapy.

In the PDX model, the study of immunomodulation was performed 18 days after cell injection preceded by 12 days of oral gavage treatment as described earlier (Fig. 6a). Both treatments alone or in combination were able to change the M1/M2 balance towards a more anti-tumoral or inflammatory response (Fig. 6b). Moreover, treatment with ibrutinib only or with the drug combination was able to diminish the frequency of PD-1-positive M2 macrophages (Fig. 6c). The frequency of SIRP $\alpha$-positive M2 macrophages was also diminished by both individual treatments, as well as the double positive M2 cells (Fig. $6 \mathrm{~d}$ and e). In this mouse model we did not observe any effect in the expression of PD-L1 by the malignant cells, while the percentage of malignant cells was also not affected by the short term treatment (Fig. $6 \mathrm{f}$ and g). Expression of CD47 by patient-derived PCNSL cells was significantly downregulated after treatment with the combination (Fig. 6h). 


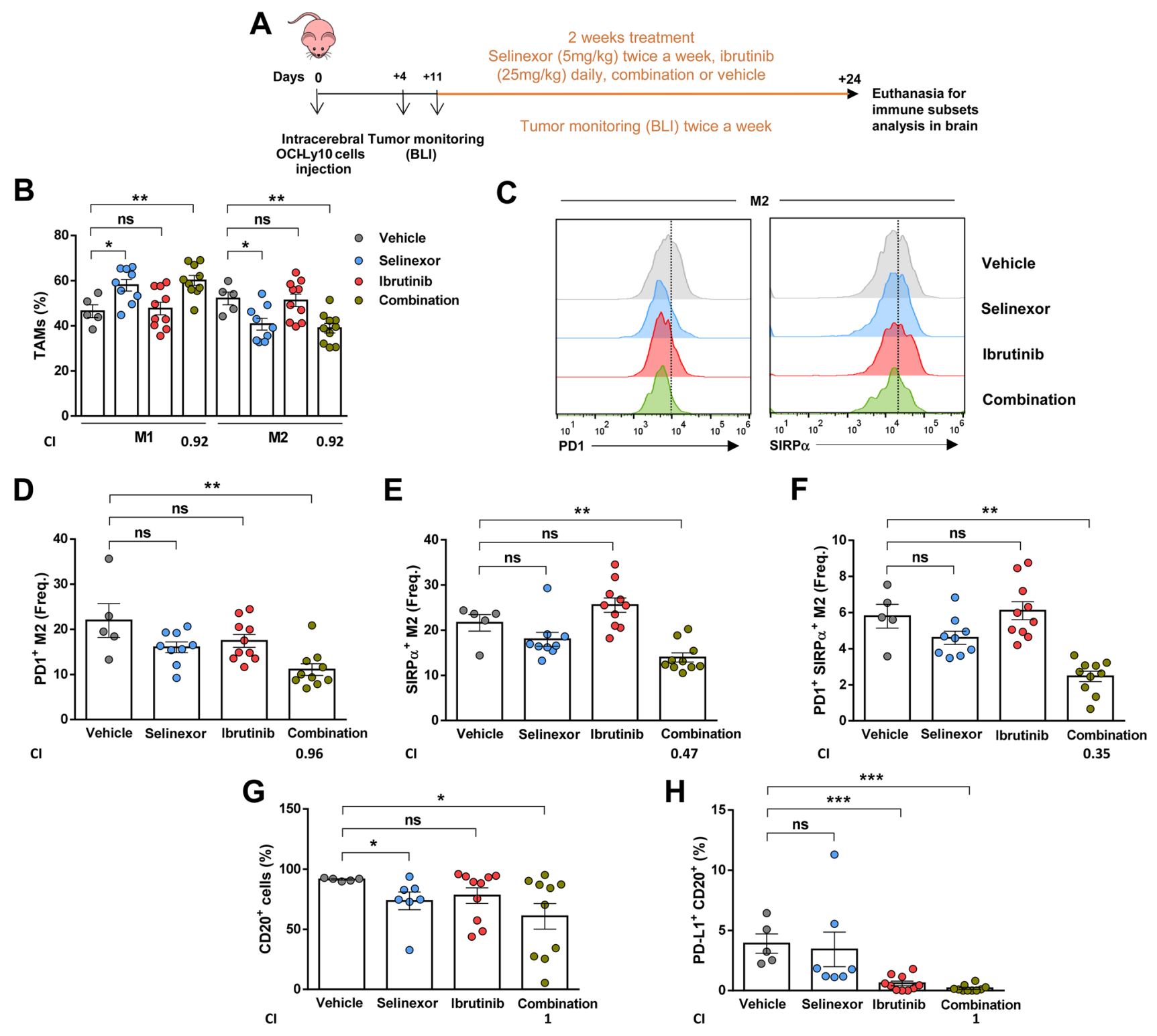

Fig. 5 Treatment with selinexor and ibrutinib favors M1-like response in tumor-associated macrophages in OCI-Ly10-derived CNS lymphomas. a Scheme representing mice treatment and monitoring. b Percentage of M1 and M2 TAMs by flow cytometry. c Histograms of $\mathrm{PD} 1^{+} \mathrm{M} 2$ and SIRP $\alpha^{+} \mathrm{M} 2$ of one representative mouse from each group. Frequency of M2 macrophages that express PD-1 (d), SIRP $\alpha$

Accordingly, CI calculations show that the combination did not improve upon individual treatments for any of the parameters except for the expression of CD47 on malignant cells. In order to identify direct immunomodulatory effects of selinexor and ibrutinib on human macrophages, we treated peripheral blood-derived macrophages in vitro with increasing doses of selinexor, ibrutinib or the combination for $30 \mathrm{~min}$ before inducing differentiation to M2 using macrophage colony-stimulating factor (M-CSF) and IL-10 (see Supplementary information for detailed (e) or co-express both markers $(\mathbf{f})$. $\mathbf{g}$ Percentage of $\mathrm{CD} 20^{+}$cells in the brains from mice treated for two weeks. $\mathbf{h}$ Percentage of CD20 $0^{+}$ malignant cells expressing PD-L1 in the different treatment groups. $(* \mathrm{P}<0.05, * * \mathrm{P}<0.01, * * * \mathrm{P}<0.001$, Mann-Whitney test. Graphs show mean $\pm \mathrm{SEM})$. $C I$ combination index, $B L I$ bioluminescence imaging

methods). M2 macrophages derived from 8 healthy donors had a mean expression of PD- 1 of $81.15 \%+/-8.8$ and mean expression of SIRP $\alpha$ of $45.53 \%+/-9.3$. Firstly, we made sure that the drugs did not affect survival of macrophages at the concentrations used (data not shown). Next, in agreement with what we observed in vivo, we observed downregulation of the expression of both PD-1 and SIRP $\alpha$ caused by individual drugs or the combination. (Supplementary Figures S4A, S4B and S4C). However, 


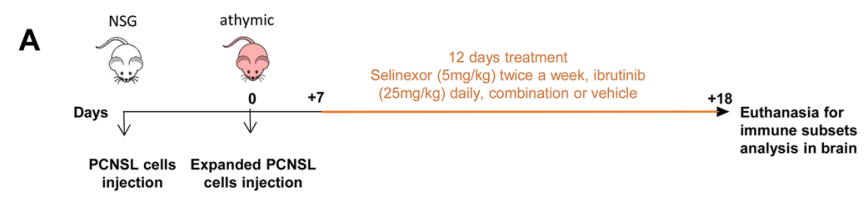

B

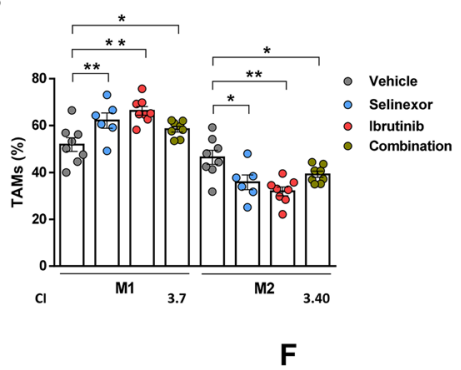

C

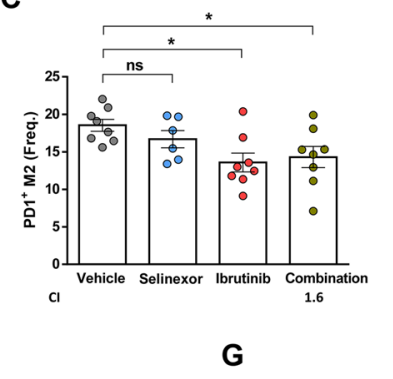

D

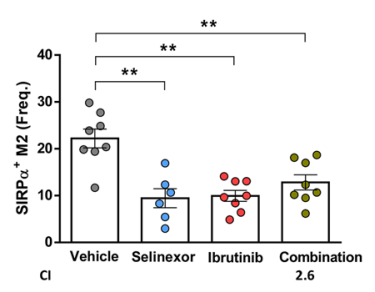

E

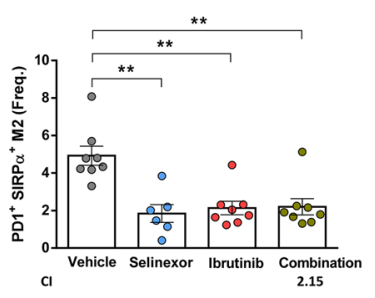

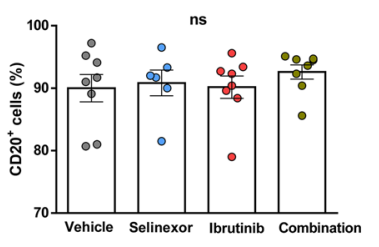
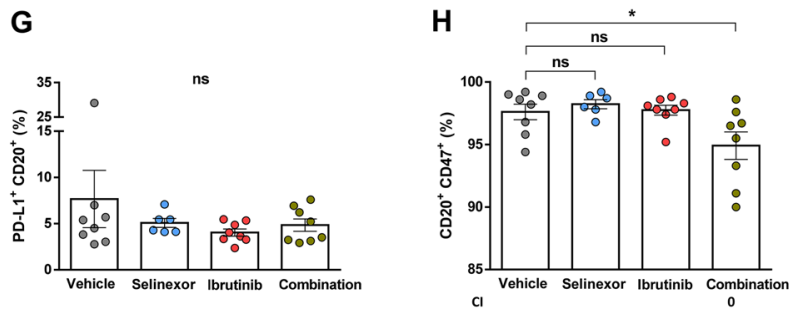

Fig. 6 Treatment with selinexor and ibrutinib favors M1-like response in tumor-associated macrophages in CNS lymphoma PDXs. a Scheme representing mice treatment and monitoring. b Percentage of M1 and M2 TAMs by flow cytometry. Frequency of M2 macrophages that express PD-1 (c), SIRP $\alpha$ (d) or co-express both markers (e). $\mathbf{f}$ Percentage of $\mathrm{CD} 20^{+}$cells in the brains from mice. $\mathbf{g}$
Percentage of malignant cells CD20 ${ }^{+}$expressing PD-L1 in the different treatment groups. Percentage of malignant cells expressing CD47 (h) and co-expressing PD-L1 and CD47 (i). (*P $<0.05$, **P $<0.01$, *** $\mathrm{P}<0.001$, Mann-Whitney test. Graphs show mean $\pm \mathrm{SEM}) . C I$ combination index

\section{Discussion}

(Supplementary Figure S4D).

Also, using the same experimental setting we analyzed the effect of selinexor, ibrutinib or the combination in interfering with M2 polarization by analyzing additional M1 and M2-like markers and IL-10 production. We found an increase in the expression of the activation and M1-like marker CD86 and a decrease in the M2-like marker CD163 as well as lower levels of PD-L1 and the anti-inflammatory cytokine IL-10 after treatment with selinexor and ibrutinib (Supplementary Figures S4E-H). However, we did not see any significant effect in the expression of CD206 or HLADR (Supplementary Figures S4I-J). In vitro modulation of additional surface markers and cytokines is consistent with the loss of pro-tumoral M2 properties after treatment with selinexor and ibrutinib.

Altogether these results indicate that the combination of selinexor and ibrutinib is able to block tumoral growth, to significantly increase the median survival of mice with PCNSL and to modulate the innate immune microenvironment towards a more anti-tumoral stage, likely reinvigorating the anti-tumoral phagocytic function of the tumor infiltrating macrophage population in vivo.
Blockage of XPO1-mediated nuclear transport using SINEs like selinexor has been shown to be an effective anti-neoplastic approach in a variety of malignancies. $[17,37,38]$ XPO1 inhibition forces nuclear localization of tumor suppressors and also interferes with additional signaling pathways, including NF- $\mathrm{KB}$ and $\mathrm{BCR}$, which are crucial for survival of malignant $\mathrm{B}$ cells in general and for PCNSL cells in particular. The clinical use of selinexor in lymphoma has been studied in a phase I trial studying patients diagnosed with relapsed/refractory NHL and a phase IIb study in patients with DLBCL [17], which has led to a recent approval by the FDA in such an adverse setting. Additionally, based on our pre-clinical experience, we recently used selinexor in a compassionate way for a patient diagnosed with DLBCL who developed an isolated CNS relapse after several lines of treatment. After a month of treatment a partial response was already observed while after 5 months of selinexor the patient remained asymptomatic and the MRI (magnetic resonance imaging) showed a complete resolution of the brain tumors [19]. Ibrutinib 
is also able to cross the $\mathrm{BBB}$ and is active against CNS lymphoma cells. In this setting, ibrutinib has been assayed alone [6,7] or in combination with chemotherapy [5], showing high response rates but relatively short remissions, while other BTK inhibitors have showed similar efficacy [39]. Based on all these data, herein we proposed to combine selinexor with ibrutinib in models of PCNSL.

Exploitation of the immune response to a neoplastic process is currently a widespread strategy to treat cancer. To achieve this, different approaches are being pursued, specially focused on harnessing the anti-tumoral capacity of $\mathrm{T}$ lymphocytes via checkpoint inhibition [40]. Intriguingly, evading a T-cell mediated immune response seems to be a common feature of PCNSL since a high percentage of cases are affected by both MHC-I loss and/or PD-L1/2 amplification [14], and the infiltration by T lymphocytes is scarce while present [9-12]. However, some immunotherapies have already shown to be effective in PCNSL, such as anti-CD20 and, more recently, anti-PD-1 therapy, with both preclinical [41] and clinical evidences, although with only information for four patients, where responses lasted a median of 15 months [42]. In agreement, anti-PD-1 is highly effective in Hodgkin's lymphoma [43] even though the expression of PD-1 on T-cells is heterogeneous and PD-L1/2 amplification and lack of MHC-I expression on tumoral cells are common, characteristics that should hamper a T-cell mediated response [44]. In this regard, a role for the innate immune system in the development of PCNSL is further supported by recent discovery of PD-1 expression in TAMs [28] and the fact that these immune cells have also been found to be suppressed by the MHC-I system in cancer cells, rendering malignant cells that downregulate MHC-I to avoid T cell surveillance exposed to macrophage phagocytosis [45]. Therefore, paralleling the few PCNSL patients treated with anti-PD-1 achieving a complete response, this effect may be related to a macrophage-mediated anti-tumoral effect after PD-1 pharmacological blockage. Supporting that, herein we describe the presence of brain PD-1-positive M2 macrophages in two orthotopic mouse models of PCNSL, including PDXs. The recognition of human malignant cells by mice macrophages has been previously demonstrated in mice models of PCNSL $[20,46]$ and other tumoral models such as colon cancer [28], pancreatic adenocarcinoma [47] and T-cell lymphoma [48]. TAMs in CNLS have been found to be supportive of the tumoral growth and related to prognosis of patients $[9,13]$. Also, indoleamine 2,3 dioxygenase (IDO) and IL-10, which may be markers of macrophage infiltration, are related to prognosis or response to immunomodulatory therapy $[9,13,49]$. The observed expression of PD-1 and SIRP $\alpha$ by innate immune cells responding to and interacting with CNS lymphoma cells in vivo indicates that their anti-tumoral effect is partially impaired but also opens the opportunity to potentially target these cells by immunotherapies that aim at potentiating the autologous anti-tumoral immune response. In this regard, it has been previously shown how immunomodulation by pomalidome in mouse models of PCNSL results in reprogramming of M2 macrophages into M1 [20]. In the clinical setting, both pomalidomide and lenalidomide are showing preliminary therapeutic activity in a phase I study in patients diagnosed with PCNSL (combined with dexamethasone) [29]. Also, lenalidomide in combination with rituximab showed significant clinical activity in relapsed/refractory PCNSL patients $[49,50]$. Combination therapies that not only directly attack the survival of malignant cells but also alter the immune function are therefore an interesting approach when aiming at achieving long lasting responses. In this regard, inhibiting BTK can have this double effect in B-cell malignancies, since BTK protein is not only involved in malignant B-cell survival but is also required for the tumor-promoting effect of macrophages $[35,36]$. Taking this into account, we hypothesized that combining ibrutinib with selinexor would also be effective in harnessing the innate immune response mediated by TAMs in PCNSL. In fact, selinexor and ibrutinib combination treatment was able to not only increase mouse survival but to shift the innate immune response towards a more inflammatory phenotype, specifically defined by downregulation of PD- 1 and SIRP $\alpha$ in M2 macrophages and increased proportion of M1 macrophages as well as modulation of additional M1 and M2-like properties consistent with loss of pro-tumoral M2 characteristics. Confirmation of these results and additional studies in the interaction of malignant cells and the immune system in PCNSL using different in vivo models, including syngeneic mice, is needed to further confirm the potential clinical value of the combination of selinexor and ibrutinib in patients diagnosed with PCNSL.

\section{Conclusions}

Our results show that selinexor blocks tumor growth and prolongs survival in a bioluminescent mouse model, while its combination with ibrutinib further increases survival. Alongside this, treatment with this combination not only had a direct cytotoxic effect in malignant cells but also favored an anti-tumoral innate immune response by shifting polarization of tumor-infiltrating macrophages toward inflammatory M1 and diminishing PD- 1 and SIRP $\alpha$ expression in the remaining tumor-promoting M2 macrophages, highlighting the pathogenic role of the innate immune microenvironment in PCNSL. Herein we provide pre-clinical evidence for the development of selinexor and ibrutinib as a new therapeutic option with cytotoxic and immunomodulatory potential for patients diagnosed with PCNSL, aiming at a durable 
response to improve the fatal prognosis of patients diagnosed with this disease.

Acknowledgements Authors want to thank Dr. Francesc Graus and Josep González from IDIBAPS, University of Barcelona, Spain and Dr. Andreu Gabarrós, from IDIBELL, University of Barcelona, Spain, for their commitment and support in this project. Authors thank the Cellex Foundation for providing research facilities and equipment.

Author contributions Designed research and supervised the work: MC and FB. Performed experiments: IJ, JC, SB, C.Pagès. JCN, JB, NP, LP. Analyzed and interpreted data: MC, FB, JS, IJ, JC. Contributed primary samples and analysis: SB, PA, JC, LE, CP, DHC, FM-R. Wrote the manuscript: IJ, JC, MC, FB. Revised the manuscript: all authors.

Funding This work was supported by research funding from the Instituto de Salud Carlos III, Fondo de Investigaciones Sanitarias (PI17/00950, M.C., PI17/00943, F.B, PI18/01392, P.A., PI16/01278, J.S) cofinanced by the European Regional Development Fund (ERDF); Fundación Asociación Española Contra el Cáncer (M.C. and P.A.) and Gilead Fellowships (GLD16/00144, GLD18/00047, F.B). M.C. holds a contract from Ministerio de Ciencia, Innovación y Universidades (RYC-2012-12018). S.B. is the recipient of a postdoctoral fellowship from Fundación Alfonso Martin Escudero.

Data availability The datasets used and/or analyzed and materials from the current study are available from the corresponding author on reasonable request.

\section{Compliance with ethical standards}

Conflicts of interest M. C. has received research funding from Karyopharm, Pharmacyclics, Roche, Arqule and AstraZeneca. F.B. has received research funding and honoraria from Roche, Celgene, Takeda, AstraZeneca, Novartis, Abbie and Janssen. J.S. is a co-founder of Mosaic Biomedicals and Northern Biologics. J.S. received grant/research support from Mosaic Biomedicals, Northern Biologics and Roche/ Glycart. PA has received honorarium for advisory and speaker faculty from Janssen, Roche, Celgene, and Abbvie. All remaining authors have declared no conflicts of interest.

Ethics approval and consent to participate All animal experiments were approved by the local Ethical Committee for the Use of Experimental Animals. The use of primary cells from patients was approved by the local Clinical Research Ethics Committee according to the principles of the Declaration of Helsinki and after obtaining written informed consent from the patient.

Informed consent Not applicable.

Open Access This article is licensed under a Creative Commons Attribution 4.0 International License, which permits use, sharing, adaptation, distribution and reproduction in any medium or format, as long as you give appropriate credit to the original author(s) and the source, provide a link to the Creative Commons licence, and indicate if changes were made. The images or other third party material in this article are included in the article's Creative Commons licence, unless indicated otherwise in a credit line to the material. If material is not included in the article's Creative Commons licence and your intended use is not permitted by statutory regulation or exceeds the permitted use, you will need to obtain permission directly from the copyright holder. To view a copy of this licence, visit http://creativecommons.org/licenses/by/4.0/.

\section{References}

1. Maher EA, Fine HA (1999) Primary CNS lymphoma. Semin Oncol 26(3):346-356

2. Camilleri-Broët $\mathrm{S}$, Crinière $\mathrm{E}$, Broët $\mathrm{P}$ et al (2006) A uniform activated B-cell-like immunophenotype might explain the poor prognosis of primary central nervous system lymphomas: analysis of 83 cases. Blood 107(1):190-196

3. Kerbauy MN, Moraes FY, Lok BH et al (2017) Challenges and opportunities in primary CNS lymphoma: a systematic review. Radiother Oncol J Eur Soc Ther Radiol Oncol 122(3):352-361

4. Nakamura T, Tateishi K, Niwa T et al (2016) Recurrent mutations of CD79B and MYD88 are the hallmark of primary central nervous system lymphomas. Neuropathol Appl Neurobiol 42(3):279-290

5. Lionakis MS, Dunleavy K, Roschewski M et al (2017) Inhibition of B cell receptor signaling by ibrutinib in primary CNS lymphoma. Cancer Cell 31(6):833-843.e5

6. Grommes C, Pastore A, Palaskas N et al (2017) Ibrutinib unmasks critical role of bruton tyrosine kinase in primary CNS lymphoma. Cancer Discov 7(9):1018-1029

7. Soussain C, Choquet S, Blonski M et al (1990) Ibrutinib monotherapy for relapse or refractory primary CNS lymphoma and primary vitreoretinal lymphoma: final analysis of the phase II "proof-of-concept" iLOC study by the Lymphoma study association (LYSA) and the French oculo-cerebral lymphoma (LOC) network. Eur J Cancer Oxf Engl 2019(117):121-130

8. Grommes C, Tang SS, Wolfe J et al (2019) Phase 1b trial of an ibrutinib-based combination therapy in recurrent/refractory CNS lymphoma. Blood 133(5):436-445

9. Nam SJ, Kim S, Kwon D et al (2018) Prognostic implications of tumor-infiltrating macrophages, M2 macrophages, regulatory T-cells, and indoleamine 2,3-dioxygenase-positive cells in primary diffuse large B-cell lymphoma of the central nervous system. Oncoimmunology 7(7):e1442164

10. He M, Zuo C, Wang J et al (2013) Prognostic significance of the aggregative perivascular growth pattern of tumor cells in primary central nervous system diffuse large B-cell lymphoma. Neuro-Oncol 15(6):727-734

11. Chang C, Lin C-H, Cheng A-L, Medeiros LJ, Chang K-C (2015) Primary central nervous system diffuse large B-cell lymphoma has poorer immune cell infiltration and prognosis than its peripheral counterpart. Histopathology 67(5):625-635

12. Venetz D, Ponzoni M, Schiraldi M et al (2010) Perivascular expression of CXCL9 and CXCL12 in primary central nervous system lymphoma: T-cell infiltration and positioning of malignant B cells. Int J Cancer 127(10):2300-2312

13. Sasayama T, Tanaka K, Mizowaki T et al (2016) Tumor-associated macrophages associate with cerebrospinal fluid interleukin-10 and survival in primary central nervous system lymphoma (PCNSL). Brain Pathol Zurich Switz 26(4):479-487

14. Chapuy B, Roemer MGM, Stewart C et al (2016) Targetable genetic features of primary testicular and primary central nervous system lymphomas. Blood 127(7):869-881

15. Etchin J, Berezovskaya A, Conway AS et al (2017) KPT-8602, a second-generation inhibitor of XPO1-mediated nuclear export, is well tolerated and highly active against AML blasts and leukemia-initiating cells. Leukemia 31(1):143-150

16. Zhong Y, El-Gamal D, Dubovsky JA et al (2014) Selinexor suppresses downstream effectors of B-cell activation, proliferation and migration in chronic lymphocytic leukemia cells. Leukemia 28(5):1158-1163

17. Kuruvilla J, Savona M, Baz R et al (2017) Selective inhibition of nuclear export with selinexor in patients with non-Hodgkin lymphoma. Blood 129(24):3175-3183 
18. Hing ZA, Mantel R, Beckwith KA et al (2015) Selinexor is effective in acquired resistance to ibrutinib and synergizes with ibrutinib in chronic lymphocytic leukemia. Blood 125(20):3128-3132

19. Bobillo S, Abrisqueta P, Carpio C, et al. 2017. Promising activity of selinexor in the treatment of a patient with refractory diffuse large B-cell lymphoma and central nervous system involvement. Haematologica.

20. Li Z, Qiu Y, Personett D et al (2013) Pomalidomide shows significant therapeutic activity against CNS lymphoma with a major impact on the tumor microenvironment in murine models. PLoS ONE 8(8):e71754

21. Yang Y, Shaffer AL 3rd, Emre NCT et al (2012) Exploiting synthetic lethality for the therapy of $\mathrm{ABC}$ diffuse large $\mathrm{B}$ cell lymphoma. Cancer Cell 21(6):723-737

22. Wilson WH, Gerecitano JF, Goy A et al (2012) The Bruton's Tyrosine Kinase (BTK) Inhibitor, Ibrutinib (PCI-32765), has preferential activity in the $\mathrm{ABC}$ subtype of relapsed/refractory de novo diffuse large B-Cell lymphoma (DLBCL): interim results of a multicenter, open-label, phase 2 study. ASH Annu Meet Abstr 120(21):686

23. Turner JG, Dawson J, Sullivan DM (2012) Nuclear export of proteins and drug resistance in cancer. Biochem Pharmacol 83(8):1021-1032

24. Rosenwald A, Wright G, Chan WC et al (2002) The use of molecular profiling to predict survival after chemotherapy for diffuse large-B-cell lymphoma. N Engl J Med 346(25):1937-1947

25. Abdul Razak AR, Mau-Soerensen M, Gabrail NY et al (2016) First-in-class, first-in-human phase i study of selinexor, a selective inhibitor of nuclear export, in patients with advanced solid tumors. J Clin Oncol Off J Am Soc Clin Oncol 34(34):4142-4150

26. de Vries R, Smit JW, Hellemans P et al (2016) Stable isotopelabelled intravenous microdose for absolute bioavailability and effect of grapefruit juice on ibrutinib in healthy adults. Br J Clin Pharmacol 81(2):235-245

27. Ponader S, Chen S-S, Buggy JJ et al (2012) The Bruton tyrosine kinase inhibitor PCI-32765 thwarts chronic lymphocytic leukemia cell survival and tissue homing in vitro and in vivo. Blood 119(5):1182-1189

28. Gordon SR, Maute RL, Dulken BW et al (2017) PD-1 expression by tumour-associated macrophages inhibits phagocytosis and tumour immunity. Nature 545(7655):495-499

29. Tun HW, Johnston PB, DeAngelis LM et al (2018) Phase 1 study of pomalidomide and dexamethasone for relapsed/refractory primary CNS or vitreoretinal lymphoma. Blood 132(21):2240-2248

30. Colonna M, Butovsky O (2017) Microglia function in the central nervous system during health and neurodegeneration. Annu Rev Immunol 35:441-468

31. Sica A, Schioppa T, Mantovani A, Allavena P. (2006). Tumourassociated macrophages are a distinct M2 polarised population promoting tumour progression: potential targets of anti-cancer therapy. Eur J Cancer Oxf Engl, 1990, 42(6): 717-727

32. Matlung HL, Szilagyi K, Barclay NA, van den Berg TK (2017) The CD47-SIRP $\alpha$ signaling axis as an innate immune checkpoint in cancer. Immunol Rev 276(1):145-164

33. Rubenstein JL, Wong VS, Kadoch C et al (2013) CXCL13 plus interleukin 10 is highly specific for the diagnosis of CNS lymphoma. Blood 121(23):4740-4748

34. Pouzoulet F, Alentorn A, Royer-Perron L et al (2019) Primary CNS lymphoma patient-derived orthotopic xenograft model capture the biological and molecular characteristics of the disease. Blood Cells Mol Dis 75:1-10

35. Nguyen P-H, Fedorchenko O, Rosen N et al (2016) LYN kinase in the tumor microenvironment is essential for the progression of chronic lymphocytic leukemia. Cancer Cell 30(4):610-622
36. Ping L, Ding N, Shi Y et al (2017) The Bruton's tyrosine kinase inhibitor ibrutinib exerts immunomodulatory effects through regulation of tumor-infiltrating macrophages. Oncotarget 8(24):39218-39229

37. Das A, Wei G, Parikh K, Liu D (2015) Selective inhibitors of nuclear export (SINE) in hematological malignancies. Exp Hematol Oncol 4:7

38. Gravina GL, Mancini A, Colapietro A et al (2017) Pharmacological treatment with inhibitors of nuclear export enhances the antitumor activity of docetaxel in human prostate cancer. Oncotarget 8(67):111225-111245

39. Nagane M, Narita Y, Mishima K, et al. (2019). Phase 1/2 study of tirabrutinib (ONO/GS-4059), a Next-Generation Bruton's Tyrosine Kinase (BTK) inhibitor, monotherapy in patients with relapsed/refractory primary central nervous system Lymphoma (PCNSL). Blood, 134(Supplement_1): 1586-1586.

40. Robert C, Ribas A, Wolchok JD et al (2014) Anti-programmeddeath-receptor-1 treatment with pembrolizumab in ipilimumabrefractory advanced melanoma: a randomised dose-comparison cohort of a phase 1 trial. Lancet Lond Engl 384(9948):1109-1117

41. Qiu Y, Li Z, Pouzoulet F et al (2018) Immune checkpoint inhibition by anti-PDCD1 (anti-PD1) monoclonal antibody has significant therapeutic activity against central nervous system lymphoma in an immunocompetent preclinical model. Br J Haematol 183(4):674-678

42. Nayak L, Iwamoto FM, LaCasce A, et al. 2017. PD-1 blockade with nivolumab in relapsed/refractory primary central nervous system and testicular lymphoma. Blood.

43. Ansell SM, Lesokhin AM, Borrello I et al (2015) PD-1 blockade with nivolumab in relapsed or refractory Hodgkin's lymphoma. N Engl J Med 372(4):311-319

44. Reichel J, Chadburn A, Rubinstein PG et al (2015) Flow sorting and exome sequencing reveal the oncogenome of primary Hodgkin and reed-sternberg cells. Blood 125(7):1061-1072

45. Barkal AA, Weiskopf K, Kao KS et al (2018) Engagement of MHC class I by the inhibitory receptor LILRB1 suppresses macrophages and is a target of cancer immunotherapy. Nat Immunol 19(1):76-84

46. Yushi Q, Li Z, Von Roemeling CA et al (2016) Osteopontin is a multi-faceted pro-tumorigenic driver for central nervous system lymphoma. Oncotarget 7(22):32156-32171

47. Massó-Vallés D, Jauset T, Serrano E et al (2015) Ibrutinib exerts potent antifibrotic and antitumor activities in mouse models of pancreatic adenocarcinoma. Cancer Res 75(8):1675-1681

48. Horwitz SM, Koch R, Porcu P et al (2018) Activity of the PI3K- $\delta$, $\gamma$ inhibitor duvelisib in a phase 1 trial and preclinical models of T-cell lymphoma. Blood 131(8):888-898

49. Rubenstein JL, Geng H, Fraser EJ et al (2018) Phase 1 investigation of lenalidomide/rituximab plus outcomes of lenalidomide maintenance in relapsed CNS lymphoma. Blood Adv 2(13):1595-1607

50. Ghesquieres H, Chevrier M, Laadhari M et al (2019) Lenalidomide in combination with intravenous rituximab (REVRI) in relapsed/refractory primary CNS lymphoma or primary intraocular lymphoma: a multicenter prospective "proof of concept" phase II study of the French Oculo-Cerebral lymphoma (LOC) Network and the Lymphoma Study Association (LYSA) $\dagger$. Ann Oncol Off J Eur Soc Med Oncol 30(4):621-628

Publisher's Note Springer Nature remains neutral with regard to jurisdictional claims in published maps and institutional affiliations. 


\section{Affiliations}

Isabel Jiménez ${ }^{1,2} \cdot$ Júlia Carabia $^{1,2} \cdot$ Sabela Bobillo ${ }^{2,3} \cdot$ Carles Palacio $^{2,3} \cdot$ Pau Abrisqueta ${ }^{2,3} \cdot$ Carlota Pagès $^{1,2}$. Juan C. Nieto ${ }^{1,2}$. Josep Castellví ${ }^{4}$. Francisco Martínez-Ricarte ${ }^{5}$. Lourdes Escoda ${ }^{6}$. Cristóbal Perla ${ }^{7}$. Dennis H. Céspedes Torrez ${ }^{7}$. Joan Boix ${ }^{1,2}$. Noelia Purroy ${ }^{1,2,10}$. Lluís Puigdefàbregas ${ }^{1,2}$. Joan Seoane ${ }^{2,8,9}$. Francesc Bosch ${ }^{2,3} \cdot$ Marta Crespo ${ }^{1,2}$

1 Experimental Hematology, Vall d'Hebron Institute of Oncology (VHIO), Vall d'Hebron Barcelona Hospital Campus, C/Natzaret, 115-117, Barcelona 08035, Spain

2 Department of Medicine, Universitat Autònoma de Barcelona, Bellaterra 08193, Spain

3 Department of Hematology, Experimental Hematology, Vall d'Hebron Hospital Universitari, Vall d'Hebron Institute of Oncology (VHIO), Vall d'Hebron Barcelona Hospital Campus, Passeig Vall d'Hebron 119-129, Barcelona 08035, Spain

4 Department of Pathology, Vall d'Hebron Hospital Universitari, Vall d'Hebron Barcelona Hospital Campus, Passeig Vall d'Hebron 119-129, Barcelona 08035, Spain

5 Department of Neurosurgery, Vall d'Hebron Hospital Universitari, Vall d'Hebron Barcelona Hospital Campus, Passeig Vall d'Hebron 119-129, Barcelona 08035, Spain
6 Department of Hematology, Hospital Universitari Joan XXIII, Tarragona, Spain

7 Department of Neurosurgery, Hospital Universitari Joan XXIII, Tarragona, Spain

8 Translational Research Program, Vall d'Hebron Institute of Oncology (VHIO), Vall d'Hebron Barcelona Hospital Campus, C/Natzaret, 115-117, 08035 Barcelona, Spain

9 Institució Catalana de Recerca I Estudis Avançats (ICREA), CIBERONC, Barcelona, Spain

10 Department of Medical Oncology, Dana-Farber Cancer Institute, Boston, Massachusetts and Broad Institute of MIT and Harvard, Cambridge, MA, UK 